\title{
Evolution and Strain Variation in BCG
}

\author{
Abdallah M. Abdallah and Marcel A. Behr
}

\begin{abstract}
BCG vaccines were derived by in vitro passage, during the years 19081921, at the Pasteur Institute of Lille. Following the distribution of stocks of BCG to vaccine production laboratories around the world, it was only a few decades before different BCG producers recognized that there were variants of BCG, likely due to different passaging conditions in the different laboratories. This ultimately led to the lyophilization of stable BCG products in the 1950s and 1960s, but not before considerable evolution of the different BCG strains had taken place. The application of contemporary research methodologies has now revealed genomic, transcriptomic and proteomic differences between BCG strains. These molecular differences in part account for phenotypic differences in vitro between BCG strains, such as their variable secretion of antigenic proteins. Yet, the relevance of BCG variability for immunization policy remains elusive. In this chapter we present an overview of what is known about BCG evolution and its resulting strain variability, and provide some speculation as to the potential relevance for a vaccine given to over 100 million newborns each year.
\end{abstract}

\section{Keywords}

Tuberculosis $\bullet$ BCG $\bullet$ Vaccine $・$ Protection $\bullet$ Strain

A.M. Abdallah $(\bowtie)$

Bioscience Core Laboratory, King Abdullah University of Science and Technology, Thuwal, Jeddah, Kingdom of Saudi Arabia

e-mail: Abdallah.abdallah@kaust.edu.sa

M.A. Behr

Department of Medicine, McGill University Health

Centre, Montreal, QC, Canada

e-mail: marcel.behr@mcgill.ca

\subsection{Introduction}

Humans have been infected with $M$. tuberculosis (Mtb) for millennia. Mtb, the intracellular pathogen that causes tuberculosis (TB), was discovered in 1882 by Robert Koch and is responsible for more human deaths than any other 
single pathogen today (Kaufmann et al. 2010; Kaufmann and Winau 2005; Ottenhoff 2009). Mycobacterium bovis Bacillus Calmette-Guérin (BCG), an attenuated strain of M. bovis, has been used as a prophylactic measure against TB for nearly a century to immunize over four billion individuals in more than 180 countries or territories (McShane 2011; Ottenhoff and Kaufmann 2012; Zwerling et al. 2011). Over $90 \%$ of children worldwide are vaccinated with BCG and more than 120 million doses of BCG are administered annually, making it the world's most widely used vaccine. Given this large denominator, it is possible to infer that there is a remarkable safety record. Unfortunately, despite the large numbers of individuals who have received BCG, in both programmatic settings and in clinical trials, the true efficacy of BCG has been difficult to understand due to many experimental variables (Griffin et al. 2001).

The parent BCG vaccine strain was derived from a virulent strain of $M$. bovis, a primary cause of TB in cattle, which is member of the $M$. $t u$ berculosis complex that mainly affects wild and domesticated mammals (see Chap. 7) (Frothingham et al. 1994; Imaeda et al. 1985). Case-control studies have shown that BCG is associated with protection against childhood disseminated TB including meningitis and miliary TB (Colditz et al. 1995; Trunz et al. 2006). However, its efficacy against pulmonary TB in adults, as measured in randomized controlled trials, has varied from no efficacy at all to as high as $80 \%$ protection (Fine 1995). It was noted as far back as 1967 that the protective efficacy of BCG against TB varies substantially between studies, showing an average risk reduction of pulmonary TB of $50 \%$, and of disseminated and meningitic forms of this disease by $70-80 \%$ (Brewer 2000; Colditz et al. 1995; Trunz et al. 2006). There are several reasons for this variation in BCG vaccine effectiveness, including differences in host populations, differences in their exposure to environmental mycobacteria and methodologic differences between the studies. In this chapter, we focus on differences between strains of BCG vaccine in use, and consider the possibility that the heterogeneity among these strains has in part contributed to the heterogeneous results from clinical trials (Behr 2002). Nowadays, several BCG strains are currently used worldwide as vaccines. Early clinical trials in indigenous groups in North America, infants in Chicago and school children in the UK demonstrated the efficacy of the vaccine and led to its distribution to several countries for worldwide application (Aronson et al. 1958; Rosenthal et al. 1961). Next to BCG, no other vaccines are available for protecting from $\mathrm{TB}$, and of the many new candidates in the pipeline none is close to market use. Therefore, it is critical to examine the product known as BCG and to ask whether evolution and strain variability in BCG may have a role in determining the capacity to provide protective immunity against TB.

\subsection{Historical Aspects of BCG}

BCG is named for Albert Calmette and Camille Guérin (Bacillus of Calmette and Guérin) who derived the original $\mathrm{BCG}$ vaccine strain from an isolate of M. bovis at the Pasteur Institute of Lille, France in the early part of the twentieth century. Since 1900, Calmette and Guérin began their research on the $M$. bovis strain, which had been isolated from the milk of a cow suffering from tuberculous mastitis by Nocard at the Pasteur Institute of Paris, France in 1902. This isolated strain of M. bovis was used by Albert Calmette and Camille Guérin to study the pathogenesis of bovine TB (Grange et al. 1983; Oettinger et al. 1999). In early studies, Calmette reported that oral inoculation of $M$. bovis resulted in pulmonary TB, through lymphatic spread from the mesenteric to the mediastinal lymph nodes. This finding presented a public health challenge, as, at the time, the predominant route of acquisition of pulmonary TB was through respiratory aerosols. To produce inocula for these experiments, Calmette and Guérin cultivated these tubercle bacilli on a glycerol-soaked potato medium, but they found that there was difficulty in the production of homogenous suspension of the bacilli and bacteria used to grow in clumps in vitro. In 
order to minimize mycobacterial clumping and make the bacteria homogenous for optimized infections, they added ox bile to the potato slices soaked in glycerol. To their surprise, they observed alterations in colony morphology within a few months of growth on this new medium, and when injected into guinea pigs, the bacilli were less virulent than the original Nocard's $M$. bovis. This fortuitous observation became source of long term project of producing a vaccine from this attenuated tubercle bacilli (Calmette 1922; Gheorghiu et al. 1983).

However, appreciating the importance of reduced virulence in terms of vaccine development, Calmette and Guérin continued the serial in vitro sub-culturing of the Nocard's M. bovis strain on potato slices soaked in ox bile and glycerol at three weekly intervals for 13 years (1908-1921), leading to total of 231 passages (Corbel et al. 2004). When administered at different doses and by different routes, the labadapted Nocard M. bovis strain was well tolerated and failed to produce progressive TB in different animal models, including guinea pigs, cows, horses, hamsters, mice, rabbits, dogs, chicken, and non-human primate (Sakula 1983). However, these cultures maintained the clumped morphology, and the same physical properties and exhibited continued immunogenicity in animal models i.e., guinea pigs, cattle, mice and chimpanzees. Furthermore, BCG vaccination protected cows against challenge with virulent $M$. bovis. These results established the safety and efficacy of BCG vaccine in experimental animals. At Guerin's suggestion, they named it Bacille Bilie CalmetteGuerin; later they omitted "Bilie" and so BCG was born (Calmette 1922).

In 1921, the BCG strain was used for the first time as a human vaccine at the request of Benjamin Weill-Halle a French pediatrician and bacteriologist. Weill-Halle wanted to protect an infant born to a mother who had died of TB a few hours after childbirth, and who was now under the care of a grandmother suffering from TB at the Charite Hospital, Paris (Bryder 1999; Sakula 1983). On July 18th, 1921, Benjamin Weill-Halle assisted by Raymond Turpin administered orally the culture of the lab-adapted Nocard's $M$. bovis strain in three doses of $2 \mathrm{mg}$ each ( $6 \mathrm{mg}$ total; $\sim 2.4 \times 10^{8}$ bacilli). On followup, there were no serious side effects, and the child did not develop any sign of TB. Based on this first anecdotal success, over the next year, additional newborns were vaccinated and no ill effects were reported. By 1924, they were able to report a series of more than 660 oral BCG vaccinations of infants (Calmette et al. 1924). For the first time, a safe and apparently effective vaccine was available for protection against human TB. The Pasteur Institute of Lille started mass-production of the $\mathrm{BCG}$ vaccine for medical applications.

As early as 1924, the original culture of BCG strain was sub-cultured and distributed to several laboratories throughout the world (Oettinger et al. 1999). These cultures were further propagated on non-synthetic culture media that varied around the world, and used for local vaccine production. This propagation of BCG on different culture media, at times following different passaging schedules, led to its diversification into a number of genetically distinct BCG sub-strains (daughter strains) (Liu et al. 2009). The first documented distribution of a daughter strain was BCG-Russia obtained in 1924 (Dubos and Pierce 1956). In the absence of lyophilisation or freezing and the production of seed-lots in 1961, BCG-Pasteur continually underwent serial passage of in vitro evolution, with daughter strains obtained directly or indirectly from the Pasteur Institute of Lille. After BCG Russia, records of BCG transfers between laboratories have shown that BCG Moreau and Japan (Tokyo-172), were obtained in 1925 (Behr and Small 1999; Obayashi 1955), Sweden in 1926 and Birkhaug in 1927 (Lind 1983; Wallgren 1928). Over the years, more than 14 sub-strains of BCG have evolved and have been used as BCG vaccine strains in different parts of the world. The main strains or seed-lots that are currently in use are: BCG Pasteur 1173 P2 (lyophilized strain in 1961 after 1173 serial passages (Gheorghiu et al. 1983)), BCG Danish 1331, BCG Glaxo 1077 (derived from the Danish strain), and BCG Moreau RDJ strains. The 


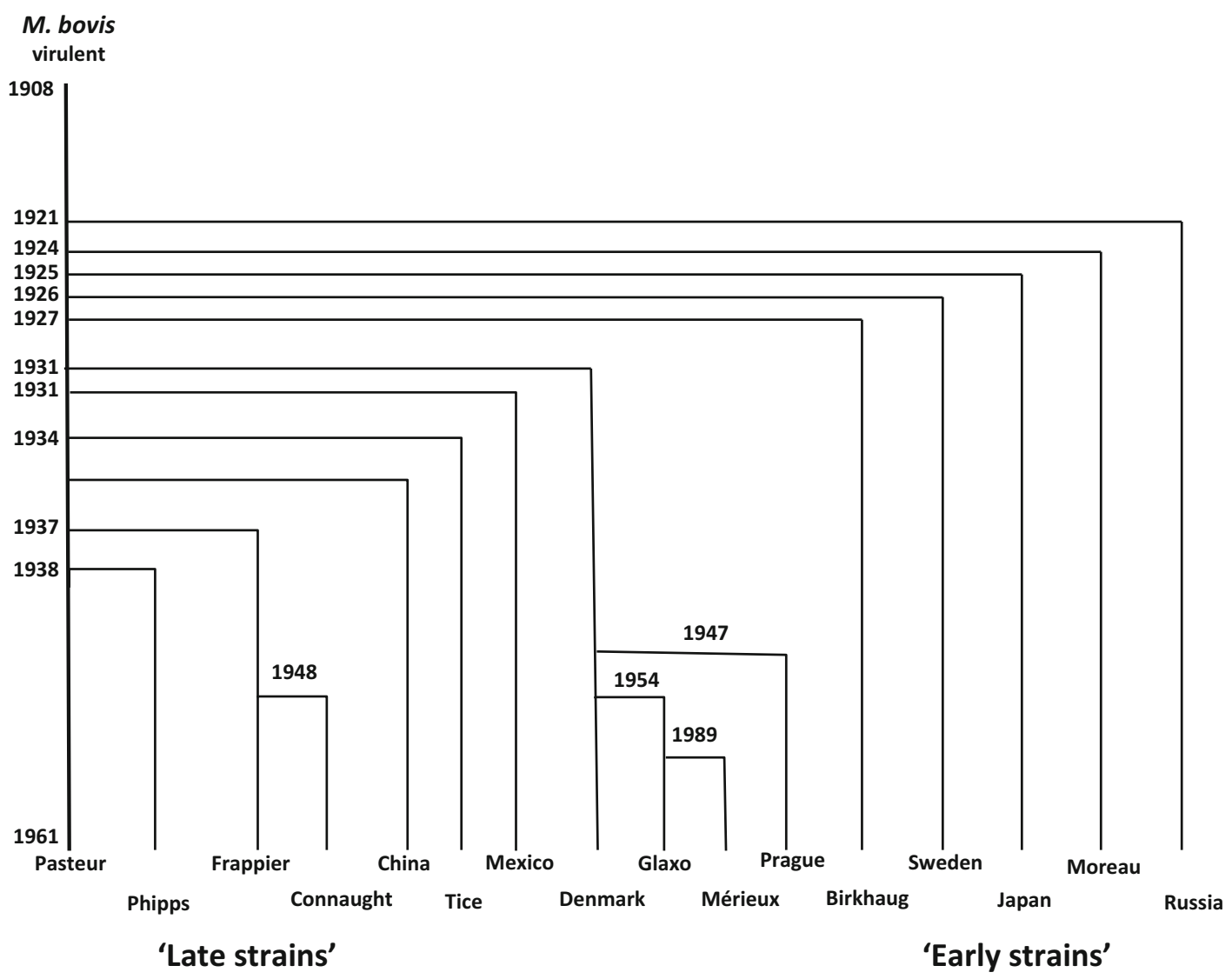

Fig. 8.1 Revised historical genealogy of BCG strain dissemination. The vertical axis represents time. The horizontal axis denotes different geographic locations of BCG propagation. Strains obtained before 1927 are

historical records of BCG dissemination separated "early strains" that were obtained in the early 1920s (BCG Russia, BCG Tokyo, BCG Moreau, BCG Sweden, BCG Birkhaug) from "late strains" that were obtained from the Pasteur Institute after 1927 (Fig. 8.1). The distinction between early strains and late strains coincides with reports of ongoing attenuation of BCG in the late 1920s (Dreyer and Vollum 1931), and is correlated with severely reduced production of the antigenic proteins MPB70, MPB83 and MPB64 in late strains (Milstien and Gibson 1990; Wiker et al. 1996). However, whether this historical distinction is critical for BCG phenotypes remains uncertain, as certain properties such as production of virulence lipids, have been inde- labelled as "early strains"; strains obtained in 1931 or later are indicated as "late strains" (Adapted from a figure by Behr et al. 1999)

pendently lost in both early and late strains (Chen et al. 2007). Furthermore, the relevance of an early versus late dichotomy in term of protective efficacy in humans cannot be ascertained, as randomized clinical trials of BCG vaccination only employed late strains.

\subsection{Phylogeny of BCG}

It is fundamental to recognize that the currently available BCG strains have undergone two phases of genomic modification. The initial phase (1908-1921) comprises the 231 in vitro passages conducted by Calmette and Guérin to produce the lab-adapted Nocard's M. bovis 
strain (the original vaccine). The second phase starts circa 1924 with widespread use and distribution of the culture of the lab-adapted Nocard's $M$. bovis strain. It ends several decades, and hundreds of passages, later (1961 for BCGPasteur 1173 P2, but different years for different daughter strains) with the establishment of frozen seed lots. Due to the initial phase, it is expected that BCG daughter strains should share the acquired particular genomic modification (BCG versus virulent $M$. bovis), whereas the second phase should give rise to additional genomic modification, specific to individual BCG strains and lineages. During the past two decades, several studies on BCG daughter strains have demonstrated changes at the genome level, using a variety of comparative genomic techniques, including subtractive hybridization, BAC libraries, spotted oligonucleotide arrays, microarray based resequencing, and whole genome sequencing. These studies have not only documented differences between BCG and $M$. bovis, but also documented additional genomic modifications that apparently were not present in the original culture obtained by Calmette and Guerin (Abdallah et al. 2015; Behr et al. 1999; Brosch et al. 2000, 2007; Gordon et al. 1999; Mahairas et al. 1996; Mostowy et al. 2003; Salamon et al. 2000). This extensive genotypic diversity in BCG daughter strains includes uncovered regions of difference (RD), single nucleotide polymorphisms (SNPs), insertion sequences (IS6110), deletions and tandem duplications.

Notably, due to the loss of the original strain of M. bovis (Nocard strain) used to derive BCG during the First World War, genomic studies have compared BCG vaccine to a variety of different $M$. bovis strains until the sequence of the $M$. bovis AF2122/97 strain was completed in 2003 (Garnier et al. 2003). As a consequence, certain differences uncovered between a particular BCG strain and the chosen M. bovis strain could represent $M$. bovis-M. bovis variants rather than BCGspecific differences. For instance, most circulating strains of $M$. bovis, including the sequenced strain AF2122/97, have only one copy of the IS6110 element. A straightforward comparison with most BCG strains might suggest that this was the ancestral state of BCG. However, a second copy of IS6110 element has been shown to be present in certain BCG daughter strains, and these were limited to BCG strains obtained before 1925 (BCGs Russia, Moreau, and Japan). While a potential explanation would be that a second IS6110 element was introduced into these different strains, the finding that they each have IS6110 element at base pair 851,592 of the $M$. tuberculosis H37Rv genome suggest the possibility that the ancestral $M$. bovis which gave rise to BCG had two copies of IS6110, with one IS6110 element deleted in 1925-1926 (Mostowy et al. 2003).

Based on the accessible data, it is now well defined which genetic characteristics are shared across all BCG daughter strains relative to $M$. bovis that may be directly involved in the attenuation of BCG, and which genetic variations are specific for only certain BCG daughter strains that may account for variation in protective efficacy and over attenuation of certain BCG daughter strains. RD1 is the example for the genomic particularities that apply to all BCG daughter strains and shown to be implicated in virulence. Deletion of RD3 and Del_Mb2377c most likely occurred during the first attenuation period (1908-1921) as these regions are absent in all BCG daughter strains (Liu et al. 2009). The variations that were observed for only certain daughter strains of BCG consist of deletions, duplications, and point mutations, and probably occurred during the following period of divergence (1921-1966). Of note, RD2 was deleted only from daughter strains derived from the BCG Pasteur strain after 1927 while nRD18 is only deleted in strains obtained after 1933.

Based on the RD1 and RD2 deletions among BCG daughter strains, two main groups of BCG have been suggested. The first group includes BCG Russia, Moreau, Japan, Sweden and Birkhaug, which were distributed from the Pasteur Institute between 1921 and 1926 and 


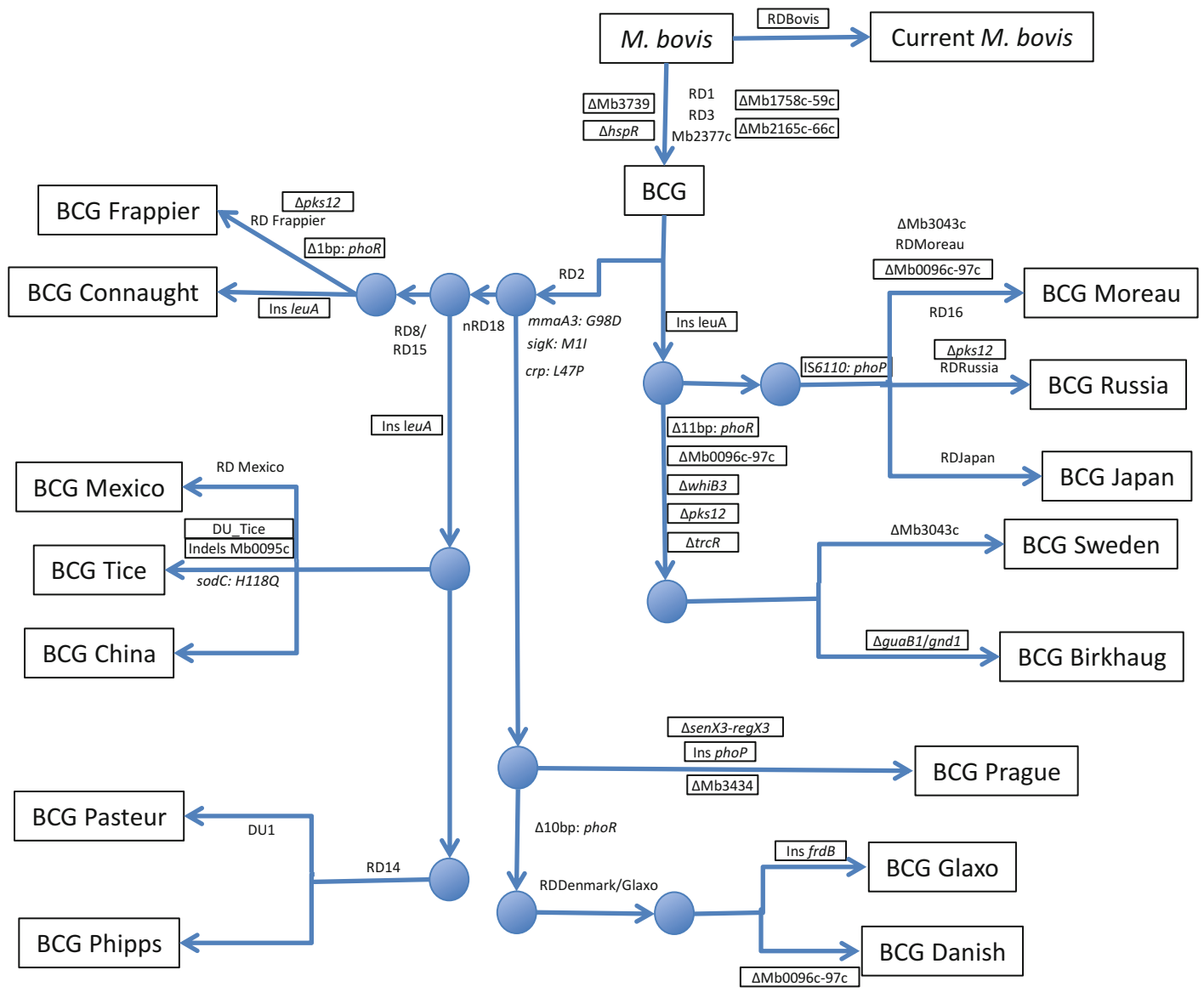

Fig. 8.2 Revised genealogy of BCG vaccines. Evolutionary scheme of BCG vaccine strains (From reference Abdallah et al. 2015), displaying the original virulent $M$. bovis ancestor strain and the subsequent series of genomic alteration including deletions of regions of difference $(R D)$, SNPs and some strain-specific insertions ('Ins') and deletions $\left({ }^{\prime} \Delta\right.$ ')

generally consistent with the historical records of BCG dissemination (Fig. 8.2).

\subsection{Molecular Evolution of BCG Daughter Strains Between 1908 and 1921} two groups have been further divided into two DU types. BCG Russia, Moreau and Japan as DU2 group I; BCG Sweden and Birkhaug as DU2 group II; BCG Prague, Glaxo, Merieux and Danish as DU2 group III and Frappier, Connaught, Tice, Mexico, China, Phipps and Pasteur as DU2 group IV (Brosch et al. 2007). Therefore, a molecular phylogeny based on this available data has been established and is Danish, Frappier, Connaught, Tice, Mexico, China, Phipps and Pasteur, and has the first group of deletions as well as the RD2 deletion. Based on tandem duplication marker, each of the above have only the RD1, RD3 and Del_Mb2377c 
to BCG attenuation has been confirmed by subsequent studies. RD1 encodes the ESX-1 protein secretion system, which is one of the five type-VII secretion systems found in the $M$. tuberculosis genome (Abdallah et al. 2007; Behr and Sherman 2007). RD1 is 9.5 kilobases (kb) in length and comprises nine genes, including the genes that encode the secreted proteins ESAT-6 (early secreted antigenic target of $6 \mathrm{kDa}$ ) and CFP-10 (culture filtrate protein of $10 \mathrm{kDa}$ ). Both of these proteins are important T-cell antigenic targets and are essential for the virulence of $M$. tuberculosis. The first experimental evidence for such contribution was obtained when the BCG Pasteur strain was complemented with the RD1 locus. The recombinant BCG strain was more virulent in severely immunodeficient mice than the BCG Pasteur strain (Pym et al. 2003). In other studies, deletion of RD1 from virulent $M$. bovis and $M$. tuberculosis strains resulted in $\triangle \mathrm{RD} 1$ mutants that were significantly attenuated for virulence in both immunocompromised and immunocompetent mice (Hsu et al. 2003; Lewis et al. 2003). In a separate approach, individual genes in the RD1 locus were identified as virulence factors for $M$. tuberculosis (Guinn et al. 2004; Hsu et al. 2003; Stanley et al. 2003). The disruption of these individual genes also resulted in the attenuation of virulence in mice. Collectively, these studies provide convincing evidence that the RD1 deletion is a key mechanism of BCG attenuation and plays a major role in virulence; although these studies did not exclude additional genetic lesions as also contributing to the loss of virulence and attenuation of BCG described by Calmette and Guerin between 1908 and 1921. Since it is common to all BCG strains, the loss of RD1 likely occurred in the initial stage of BCG attenuation.

A recent comparative genome analysis of multiple BCG daughter strains using whole genome sequencing has uncovered a 103 bp deletion present across all BCG daughter strains, and which eliminates the distal end of $h s p R$ (Abdallah et al. 2015). This gene is involved in transcriptional regulation (repression) of heat shock proteins and is known to impact virulence. The $h s p R$ locus activates a subset of the heat-shock general stress response upon macrophage invasion (Stewart et al. 2002), and is necessary in the persistent phase since strains with an hspR deletion $(\Delta \mathrm{hspR})$ exhibit attenuated growth in chronic infection (Stewart et al. 2001). Furthermore, the same study showed that a duplication of $2900 \mathrm{bp}$ segments spanning the region between 1,276,501 and $1,279,400$ base pairs (M. bovis coordinates) in all BCG daughter strains (Abdallah et al. 2015).

Although the most attributed reason for the primary attenuation of BCG compared to $M$. bovis is the loss of the RD1 locus, complementation of BCG with this region does not fully restore virulence to wild-type levels (Pym et al. 2003), and the RD1 deletion mutant of M. tuberculosis is still more virulent than BCG in long-term murine infection experiments (Sherman et al. 2004). This leads to speculation that additional genetic lesions that have occurred in BCG also contribute to its attenuation. Whole genome sequence comparison revealed 736 single nucleotide polymorphisms (SNPs) between BCG-Pasteur 1173P2 and M. bovis AF2122/97 (Brosch et al. 2007). Recent comprehensive studies of SNPs between $M$. bovis and BCG daughter strains revealed two types of SNPs that could also play a role in the early evolution of BCG (Abdallah et al. 2015, Pelayo et al. 2009). Most are common to the entire BCG lineage, but some SNPs are specific to individual BCG strains. This suggests that loss of virulence during the initial 231 in vitro passages of BCG involved both the loss of RD1 and other mutations (the aforementioned deletion and duplication, plus SNPs), which together likely contributed to the attenuation of virulence during the derivation of BCG.

Of the SNPs that are common to all BCG daughter strains, some have been explored in greater depth, but further study is required. For example, SNPs in genes predicted to be involved in glycerol catabolism were identified in two genes, glpK (codon 191) and $p y k A$ (codon 220). glpK encodes glycerol kinase, the enzyme which catalyzes the MgATP-dependent phosphorylation of glycerol to yield sn-glycerol 3-phosphate, the rate limiting step in glycerol 
utilization in E. coli (Zwaig et al. 1970). Remarkably, this SNP is present in M. bovis AF2122/97 and causes a frameshift in the glpK gene at codon 191, leading to a truncated coding sequence (Garnier et al. 2003). Noteworthy, the $g l p K$ frameshift is not evident in the BCG daughter strains or in the M. bovis AN5 strain, strains that can grow on glycerol as the sole carbon source (Keating et al. 2005). The second SNP is a nucleotide substitution in $p y k A$, the gene that encodes pyruvate kinase, an enzyme that catalyzes the final step in glycolysis, the conversion of phosphoenolpyruvate to pyruvate. The pykA SNP results in the substitution of glutamic acid 220 by aspartic acid and renders pyruvate kinase nonfunctional. This highly conserved glutamic acid residue is predicted to play an important role in the active site of pyruvate kinase and has been associated with cofactor $(\mathrm{Mg}++)$ and substrate (ADP/ATP, PEP) binding (Munoz and Ponce 2003). As with the glpK SNP, the pykA mutation does not occur in the BCG daughter strains or M. bovis AN5 but is present in M. bovis AF2122/97 and was suggested to account for inability of $M$. bovis to grow on glycerol as a sole carbon source (Keating et al. 2005). This suggests that progenitors of BCG daughter strains (Nocard's M. bovis) and $M$. bovis AN5 strain had an in-frame $g l p K$ and pykA coding sequence.

Another study implicated one of the first BCG polymorphism described in Mb3700, a gene encoding a transcriptional regulator of the cyclic AMP (cAMP) receptor protein (CRP)- fumarate and nitrate reduction regulator (FNR) family that could affect the DNA binding activity of this putative global transcriptional regulator and therefore, could contribute to the attenuation of BCG strains. Further studies demonstrated that this point mutation resulting in a base substitution of glutamic acid to lysine (E178K) present in all BCG daughter strains examined altered DNA binding of CRP to target sites as well as global gene expression, without playing any role in the attenuation of BCG (Bai et al. 2007; Hunt et al. 2008). Although these lesions have not been directly associated with the original attenuation of $\mathrm{BCG}$, it remains possible that the accumulation of multiple lesions, including SNPs, between 1908 and 1921 may have resulted in a complex effect that is yet to be fully resolved.

\subsection{Molecular Evolution of BCG Daughter Strains After 1924}

Dissemination of BCG from Pasteur Institute to various parts of the world began in 1924. Subsequent to the original derivation of BCG, strains were passaged in a variety of non-synthetic media, based on variable growth factors such as the local potatoes. As these laboratory conditions were not uniform, it was perhaps not surprising that vaccine producers recognized the emergence of BCG daughter strains with distinct morphological, biochemical and immunological phenotypic properties by the 1940 s and 1950 s. Thanks to genomic studies, it is now known that different BCG strains differ both from the original BCG of 1921 and from each other, due to deletions, SNP and duplications. Thus there is no "ancestral" BCG in existence. For instance, BCG Pasteur may be considered the reference strain of BCG vaccines, but the sequenced BCG Pasteur 1173 is separated from the BCG of 1921 by a number of genetics events (e.g., deletion of IS6110, RD2, nRD18, and RD14; SNPs in $m m A 3, \operatorname{sig} K$, and $c r p \_L 47 P$; duplication of DU1) (Fig. 8.2). Evidence to suggest a closer relationship between BCG Russia and the original BCG progenitor strain emerged from the discovery that BCG Russia is a natural recA mutant (Keller et al. 2008). Because a RecA mutation might result in fewer mutation, it was speculated that this mutation might have kept BCG Russia in a state 'closer' to the 1921 ancestor of all BCG strains. Indeed, upon determining the complete genome sequence of a panel of BCG strains, it was shown that BCG Russia contains fewer SNPs and deletions compared to other BCG strains (Abdallah et al. 2015).

A critical divergence in the evolution of BCG was the loss of the $10.8 \mathrm{~kb}$ region of $\mathrm{RD} 2$ during the ongoing propagation of BCG between 1927 and 1931, a time that coincides with reports of the ongoing attenuation of the vaccine. This 
led to "early" (RD2 present) and "late" (RD2 absent) BCG strains. The role of RD2-associated virulence was evaluated by a targeted knockout in the M. tuberculosis reference strain $\mathrm{H} 37 \mathrm{Rv}$, which showed that the deletion mutant was attenuated in murine models of infection (Kozak et al. 2011). Moreover, some of the genes encoded by RD2 stand out as candidates for the ongoing attenuation of BCG daughter strains in the laboratory. The deletion of RD2, which contains the gene $m p b 64$ and encodes the antigenic protein MPB64, accounts for the lack of MPB64 in the late strains. Complementation of the BCG daughter strain Pasteur with $m p b 64$ gene improved the immunogenicity of the vaccine strain but did not improve protection against pulmonary TB (Kozak and Behr 2011).

In addition to RD2, several other genomic polymorphisms that are common to all strains obtained from the Pasteur Institute after 1927 have been investigated, including point mutations that may have contributed to the later evolution of BCG. For instance, protein antigens MPB70 and MPB83 are found to be at high levels in early strains prior to 1927 (acquired before 1927) but are present only in trace quantities in late BCG strains (Milstien and Gibson 1990). Follow-up studies using immunoblot and quantitative reverse transcription polymerase chain reaction (RT-PCR) separated BCG into high- and low-producing strains, and determined that transcription of the antigen-encoding genes, $m p b 70$ and $m p b 83$, follows the same strain pattern with mRNA levels reduced over 50-fold in low-producing strains (Charlet et al. 2005). Using transcriptomic analysis, Charlet et al. identified two regions of the genome that had dysregulated gene expression between high-producers and low-producers: the set of genes including $m p b 70$ and $m p b 83$, and a distant set of genes including the gene coding for Sigma Factor K. DNA sequence analysis showed a point mutation in the $\operatorname{Sig} K$ gene, leading to gene complementation studies that formally implicated this mutation in the decreased expression of MPB70 and MPB83 in the later strains (Charlet et al. 2005).

Strains obtained from the Pasteur Institute prior to 1927 produced methoxymycolates, a sub- class of cell wall mycolic acids in vitro but those obtained later could not synthesize methoxymycolates. This phenotype has been attributed to a point mutation in the mmaA3 gene, which encodes methoxy mycolic acid synthase 3 and is responsible for O-methylation of hydroxymycolate precursors to form methoxymycolic acids. This base substitution at position 293 in mmaA3 results in an amino acid change from glycine to aspartic acid and inhibits methoxymycolates production (Behr et al. 2000). Also, it has been postulated that this SNP leads to low-level isoniazid (INH) resistance (Abdallah et al. 2015), as INH is known to inhibit the synthesis of $\alpha$-mycolate, methoxymycolate and $\beta$-mycolate (Takayama et al. 1972). Intriguingly, the loss of methoxymycolate appears to have no impact on the virulence of late BCG strains (Belley et al. 2004). Furthermore, two predicted regulators have been disrupted during the serial passage of BCG strains. The sigI gene (possible alternate RNA polymerase sigma factor SigI) encoded in nRD18 is missing from BCG strains obtained after 1933. Additionally, RD14, which encodes Mb1802 and has been annotated as a probable transcriptional regulatory protein, is deleted from BCG-Pasteur (Behr et al. 1999) and some strains of BCG-Phipps (Abdallah et al. 2015); this predicted role as a transcriptional regulator was subsequently confirmed with promoter fusion assays (Alexander and Behr 2007). Finally, Mb3439c, a gene encoded in RD16 and also annotated as a possible transcriptional regulator has been disrupted by independent deletions in BCG-Moreau and BCG-Japan. The loss of regulatory genes from a number of different BCG strains argues that mutation of regulatory genes can be tolerated during conditions of laboratory growth.

BCG-Japan, BCG-Moreau and BCG-Glaxo do not produce the lipid virulence factors phthiocerol dimycocerosates (PDIMs) and phenolic glycolipids (PGLs), and were found to be naturally deficient in PDIMs and PGLs, whereas the other BCG strains do produce these lipids (Chen et al. 2007). Because some strains of BCG have been associated with higher rates of adverse effects, such as disseminated BCGosis, investigators have queried whether the presence/absence 
of these virulence factors might in part explain the variable rates of these events. Indeed, the loss of these lipids has been shown to correlate with the superior safety records of these strains in clinical studies (Chen et al. 2007; Lotte et al. 1984). Moreover, deletion of PIDMs/PGLs from BCG Pasteur reduces its virulence and protective efficacy (Tran et al. 2016). Intriguingly, variation in PDIM and PGL production does not coincide with the genealogy of BCG strains (Abdallah et al. 2015; Brosch et al. 2007), suggesting that this particular phenotype has emerged multiple times and by multiple mechanisms. Indeed, in BCG-Moreau, the PDIM and PGL defect is likely due to a 975-bp deletion that affects fadD26 and ppsA (Leung et al. 2008), which are members of the PDIMs and PGLs biosynthetic locus (Azad et al. 1997, Leung et al. 2008). Similarly, a point mutation in ppsA is responsible for the lack of PDIMs/PGLs in BCG-Japan (Naka et al. 2011). However, this region is intact in BCGGlaxo (Leung et al. 2008), indicating that the PDIM/PGL defect in BCG-Glaxo is caused by other, currently unknown genetic lesions. Together, this suggests that these three BCG strains independently acquired mutations in PDIM/PGL biosynthesis that contributed to their further attenuation.

Considering its prominent role in virulence, it may not be surprising that BCG strains exhibit a number of genetic polymorphism in the phoP-phoR locus (Leung et al. 2008), a twocomponent system known to regulate the expression of multiple genes, including some wellestablished T-cell antigens (Walters et al. 2006). Of note, a frame-shift mutation within the phoP gene of BCG-Prague eliminates the majority of C-terminal DNA binding domain, and makes this strain a natural phoP mutant (Gupta et al. 2006; Sinha et al. 2008; Wang et al. 2007), and it is possible that this could account for this strain's reported low immunogenicity (Ladefoged et al. 1976; Vallishayee et al. 1974). Furthermore, three early strains as mentioned earlier, BCG-Russia, BCG-Japan and BCG-Moreau have an IS6110 insertion in the promoter region of phoP (Leung et al. 2008), which may eliminate the auto-repression regulatory mechanism of this two component system (Gupta et al. 2006). BCGSweden and BCG-Birkhaug contain a deletion that truncates the C-terminal of PhoR (Leung et al. 2008). In BCG-Danish, BCG-Glaxo and BCG-Frappier, frame-shift mutations in the $p h o R$ gene abolish the PhoR protein (Leung et al. 2008).

Unlike other BCG strains, two closely related BCG strains, BCG-Sweden and BCG-Birkhaug, contain deletions in whiB3, a transcriptional regulator implicated in virulence of M. bovis (Steyn et al. 2002) and M. tuberculosis (Singh et al. 2009), in $p k s 12$, a polyketide synthase that necessary for CD1c-mediated T-cell response, which may affect the immunogenicity of the vaccine (Matsunaga et al. 2004), and in $\operatorname{trcR}$, the response regulator of the TrcR-TrcS two-component system that controls expression of the $\operatorname{trcRS}$ operon, including $m m p S 5 / m m p L 5$ transporter and $b f r B$ bacteroferrin (Wernisch et al. 2003). Intriguingly, deletion of $\operatorname{trc} S$ from $M$. tuberculosis produced a hypervirulent phenotype in SCID mice (Parish et al. 2003), suggesting a role for this gene in the attenuation of BCG. However, these deletions are not present in other BCG strains and therefor distinguish the BCG-Sweden and BCGBirkhaug lineage from other early strains (e.g., BCG-Russia, BCG-Japan and BCG-Moreau).

Comparative genomics has also uncovered two large tandem duplications, DU1 and DU2 of 29-kb and 36-kb, respectively, in BCG-Pasteur (Brosch et al. 2000, 2007). These seem to have arisen independently, as their presence and/or their size varies between the different BCG daughter strains. While DU1 appears to be restricted to BCG-Pasteur, DU2 has detected in all BCG daughter strains tested so far but at variable size (Abdallah et al. 2015; Brosch et al. 2007). Interestingly, DU1 contains the oriC locus, the site of chromosomal origin of replication, indicating that BCG-Pasteur is diploid for oriC, and several key genes involved in replication initiation and cell division machinery (Brosch et al. 2000). For DU2, the tandem duplication resulted in diploidy for 30 genes, for which probable functions are known. These include aroA, a key enzyme in aromatic amino acid biosynthesis, and the coding 
sequences for variety of regulatory proteins that could exert pleiotropic effects, including a histidine kinase $a s n C$ and tet $R$ homologues, whiBl and sigH, a sigma factor implicated in the heat shock response (Fernandes et al. 1999). Chromosomal duplications are a common evolutionary response in bacteria exposed to different selection pressures in the laboratory and presumably in nature, as they provide a means for increasing gene dosage and for generating novel functions from potential gene fusion events at duplication endpoints. They also represent a source of redundant DNA for divergence. As such, the presence of DU1 and DU2 suggests that the process of tandem duplications in BCG is ongoing and remains a potent source of genome dynamics. However, the potential role of these duplication events on the immunogenicity of BCG strains remains to be explored. Other duplications specific to certain BCG strains have also been uncovered, such as a 22-kb duplication present only in BCG Tice (DUTice) (Leung et al. 2008). DU-Tice contains the entire ESX-5 secretion system, which is present only in pathogenic mycobacteria (Abdallah et al. 2007), and has been shown to be responsible for the transport of cell envelope proteins that are required for nutrient uptake (Ates et al. 2015), and directly or indirectly modulate the human macrophages response (Abdallah et al. 2008). Interestingly, sequencing of BCGChina repositioned this strain from the DU2III group into the DU2-IV group that includes BCG-Pasteur, BCG-Phipps, BCG-Tice, BCGMexico, BCG-Connaught and BCG-Frappier. This analysis revealed that BCG-China was not originally derived from BCG-Danish, as previously thought, and that this inconsistency was likely attributed to multiple circulating strains of "BCG China" (Abdallah et al. 2015).

\subsection{Implications for BCG Immunization}

BCG vaccines are given to over a hundred millions of newborns each year, with the goal of safely preventing TB disease. The ideal profile of such a vaccine should be: (1) prevents disease, (2) rarely causes progressive vaccine-associated infection, and (3) if vaccine infection occurs, this should be readily treatable. At present, it is unclear, despite all known from genomic studies, whether a particular BCG is better, or less desirable, at addressing the aforementioned criteria.

For protective efficacy, a lingering question is whether early strains, which produce antigens such as MPB64, MPB70 and MPB83, are more likely to protect against TB. However, there have been no randomized trials that have competed an early strain against a late strain of BCG; in fact, there have been no randomized controlled trials whatsoever of the early BCG strains. For safety, there have been reports that the early strains have been associated with a greater risk of vaccineassociated infection (also known as BCG-osis). However, these reports were generally confined to before-and-after studies in countries with a change of vaccine strain for political reasons. Formal demonstration that early BCG strains are more virulent, and therefore, a greater risk in immunization schedules, is lacking. Finally, for treatment of rare cases of BCG-osis, it is worth noting that all BCG strains are resistant to pyrazinamide, as this is a genetic feature of $M$. bovis (Huard et al. 2006), and that some strains are apparently resistant to isoniazid (Abdallah et al. 2015; Kolibab et al. 2011). The degree of isoniazid resistance is considered low-level, such that some consider this not to be a clinical issue (Arend and van Soolingen 2011). In addition, they are also resistant to cycloserine, partly due to the G122S mutation in $c y c A$ (Chen et al. 2012). Interestingly, expression of a functional copy of $c y c A$ from M. tuberculosis or M. bovis increased the susceptibility of BCG Pasteur to dcycloserine, albeit not to the levels of $M$. bovis or M. tuberculosis (Chen et al. 2012), suggesting that other genetic lesions may contribute to BCG resistance to d-cycloserine. Nonetheless, it is not intuitive that we should be giving a live attenuated vaccine to millions of newborns, knowing that some of these vaccines are inherently resistant to two of the four antibiotics used to treat TB. Further study should aim to formally identify the mutation(s) responsible for isoniazid resistance, 
as there could be policy implications in knowing which strains might not be ideal for vaccination purposes.

Finally, BCG is used not only to prevent TB, but also as an immunotherapeutic for bladder cancer. The lessons of BCG evolution and strain variability have yet to affect the choice of BCG strains for this indication, yet, many parallels can be imagined. How does BCG work in bladder cancer? What are the adverse effects? How is BCG-osis treated in an elderly, debilitated patient suffering from a malignancy? Further study of the BCG strain family, particularly with the goal of linking genotype to clinically-meaningful phenotype, is required, to find the best use of these bacteria given to us by the work of Calmette and Guerin.

\subsection{Conclusion}

More than 100 years ago, Albert Calmette and Camille Guérin began their research for an antiTB vaccine, which lasted 13 years and led to one of the most widely used vaccines in human history. Despite a century of investigation, the BCG vaccine continues to be controversial and remains the only vaccine for the prevention of TB. Shaped by human history, BCG has also evolved to the various daughter strains recognized today. These evolved strains differ from each other and from the original BCG first used in 1921, both genetically and phenotypically, such that these changes may translate into variable vaccine properties, including protective efficacy, tuberculin reactivity and propensity for adverse effects. Remarkably, the current collection of BCG comprises various natural mutants of established virulence factors. Continuing studies on the molecular factors that impact properties of BCG vaccine strains would shed light on the specific differences among BCG strains and further our understanding of the mechanisms of attenuation specific to each lineage. This would be useful in further delineating BCG strains into phenotypic and genetic categories that could mediate their protective efficacy.

\section{References}

Abdallah AM, Gey Van pittius NC, Champion PA, Cox J, Luirink J, Vandenbroucke-Grauls CM, Appelmelk BJ, Bitter W (2007) Type VII secretion - mycobacteria show the way. Nat Rev Microbiol 5:883-891

Abdallah AM, Savage ND, Van zon M, Wilson L, Vandenbroucke-Grauls CM, Van Der Wel NN, Ottenhoff TH, Bitter W (2008) The ESX-5 secretion system of Mycobacterium marinum modulates the macrophage response. J Immunol 181:7166-7175

Abdallah AM, Hill-Cawthorne GA, Otto TD, Coll F, Guerra-Assuncao JA, Gao G, Naeem R, Ansari H, Malas TB, ADROUB SA, Verboom T, Ummels R, Zhang H, Panigrahi AK, Mcnerney R, Brosch R, Clark TG, Behr MA, Bitter W, Pain A (2015) Genomic expression catalogue of a global collection of BCG vaccine strains show evidence for highly diverged metabolic and cell-wall adaptations. Sci Rep 5: 15443

Alexander DC, Behr MA (2007) Rv1773 is a transcriptional repressor deleted from BCG-Pasteur. Tuberculosis (Edinb) 87:421-425

Arend SM, Van Soolingen D (2011) Editor's choice: editorial commentary: low level INH-resistant BCG: a sheep in wolf's clothing? Clin Infect Dis 52:89-93

Aronson JD, Aronson CF, Taylor HC (1958) A twentyyear appraisal of BCG vaccination in the control of tuberculosis. AMA Arch Intern Med 101:881-893

Ates LS, Ummels R, Commandeur S, Van de Weerd R, Sparrius M, Weerdenburg E, Alber M, Kalscheuer R, Piersma SR, Abdallah AM, Abd el Ghany M, AbdelHaleem AM, Pain A, Jimenez CR, Bitter W, Houben EN (2015) Essential role of the ESX-5 secretion system in outer membrane permeability of pathogenic mycobacteria. PLoS Genet 11:e1005190

Azad AK, Sirakova TD, Fernandes ND, Kolattukudy PE (1997) Gene knockout reveals a novel gene cluster for the synthesis of a class of cell wall lipids unique to pathogenic mycobacteria. J Biol Chem 272:1674116745

Bai G, Gazdik MA, Schaak DD, Mcdonough KA (2007) The Mycobacterium bovis BCG cyclic AMP receptorlike protein is a functional DNA binding protein in vitro and in vivo, but its activity differs from that of its $\mathrm{M}$. tuberculosis ortholog, Rv3676. Infect Immun 75:55095517

Behr MA (2002) BCG - different strains, different vaccines? Lancet Infect Dis 2:86-92

Behr MA, Sherman DR (2007) Mycobacterial virulence and specialized secretion: same story, different ending. Nat Med 13:286-287

Behr MA, Small PM (1999) A historical and molecular phylogeny of BCG strains. Vaccine 17:915-922

Behr MA, Wilson MA, Gill WP, Salamon H, Schoolnik GK, Rane S, Small PM (1999) Comparative genomics of BCG vaccines by whole-genome DNA microarray. Science 284:1520-1523 
Behr MA, Schroeder BG, Brinkman JN, Slayden RA, Barry CE 3rd (2000) A point mutation in the mma3 gene is responsible for impaired methoxymycolic acid production in Mycobacterium bovis BCG strains obtained after 1927. J Bacteriol 182:3394-3399

Belley A, Alexander D, Di Pietrantonio T, Girard M, Jones J, Schurr E, Liu J, Sherman DR, Behr MA (2004) Impact of methoxymycolic acid production by Mycobacterium bovis BCG vaccines. Infect Immun 72:2803-2809

Brewer TF (2000) Preventing tuberculosis with Bacillus Calmette-Guerin vaccine: a meta-analysis of the literature. Clin Infect Dis 31(Suppl 3):S64-S67

Brosch R, Gordon SV, Buchrieser C, Pym AS, Garnier T, Cole ST (2000) Comparative genomics uncovers large tandem chromosomal duplications in Mycobacterium bovis BCG Pasteur. Yeast 17:111-123

Brosch R, Gordon SV, Garnier T, Eiglmeier K, Frigui W, VALENTI P, Dos Santos S, Duthoy S, Lacroix C, Garcia-Pelayo C, Inwald JK, Golby P, Garcia JN, Hewinson RG, Behr MA, Quail MA, Churcher C, Barrell BG, Parkhill J, Cole ST (2007) Genome plasticity of BCG and impact on vaccine efficacy. Proc Natl Acad Sci U S A 104:5596-5601

Bryder L (1999) 'We shall not find salvation in inoculation': BCG vaccination in Scandinavia, Britain and the USA, 1921-1960. Soc Sci Med 49:1157-1167

Calmette A (1922) L'ninfection bacillaire et la tuberculose chez l'homme et chez les animaux

Calmette A, Guerin C, Weill-Halle B (1924) Essai d'immunisation contre l'infection tuberculeuse. Bull Acad Med Paris 91:787-796

Charlet D, Mostowy S, Alexander D, Sit L, Wiker HG, Behr MA (2005) Reduced expression of antigenic proteins MPB70 and MPB83 in Mycobacterium bovis BCG strains due to a start codon mutation in sigK. Mol Microbiol 56:1302-1313

Chen JM, Islam ST, Ren H, Liu J (2007) Differential productions of lipid virulence factors among BCG vaccine strains and implications on BCG safety. Vaccine 25:8114-8122

Chen JM, Uplekar S, Gordon SV, Cole ST (2012) A point mutation in cycA partially contributes to the Dcycloserine resistance trait of Mycobacterium bovis BCG vaccine strains. PLoS One 7:e43467

Colditz GA, Berkey CS, Mosteller F, Brewer TF, Wilson ME, Burdick E, Fineberg HV (1995) The efficacy of bacillus Calmette-Guerin vaccination of newborns and infants in the prevention of tuberculosis: meta-analyses of the published literature. Pediatrics 96:29-35

Corbel MJ, Fruth U, Griffiths E, Knezevic I (2004) Report on a WHO consultation on the characterisation of BCG strains, Imperial College, London 15-16 December 2003. Vaccine 22:2675-2680

Dreyer G, Vollum RL (1931) Mutation and pathogenicity experiments with BC G. Lancet 1:9-15

Dubos RJ, Pierce CH (1956) Differential characteristics in vitro and in vivo of several substrains of BCG. IV. Immunizing effectiveness. Am Rev Tuberc 74:699-717
Fernandes ND, Wu QL, Kong D, Puyang X, Garg S, Husson RN (1999) A mycobacterial extracytoplasmic sigma factor involved in survival following heat shock and oxidative stress. J Bacteriol 181:4266-4274

Fine PE (1995) Variation in protection by BCG: implications of and for heterologous immunity. Lancet 346:1339-1345

Frothingham R, Hills HG, Wilson KH (1994) Extensive DNA sequence conservation throughout the Mycobacterium tuberculosis complex. J Clin Microbiol 32:1639-1643

Garnier T, Eiglmeier K, Camus JC, Medina N, Mansoor H, Pryor M, Duthoy S, Grondin S, Lacroix C, Monsempe C, Simon S, Harris B, Atkin R, Doggett J, Mayes R, Keating L, Wheeler PR, Parkhill J, Barrell BG, Cole ST, Gordon SV, Hewinson RG (2003) The complete genome sequence of Mycobacterium bovis. Proc Natl Acad Sci U S A 100:7877-7882

Gheorghiu M, Augier J, Lagrange PH (1983) Maintenance and control of the French Bcg strain 1173-P2 (primary and secondary seed-lots). Bull Inst Pasteur 81: 281-288

Gordon SV, Brosch R, Billault A, Garnier T, Eiglmeier K, Cole ST (1999) Identification of variable regions in the genomes of tubercle bacilli using bacterial artificial chromosome arrays. Mol Microbiol 32:643-655

Grange JM, Gibson J, Osborn TW, Collins CH, Yates MD (1983) What is BCG? Tubercle 64:129-139

Griffin JF, Chinn DN, Rodgers CR, Mackintosh CG (2001) Optimal models to evaluate the protective efficacy of tuberculosis vaccines. Tuberculosis (Edinb) 81:133-139

Guinn KM, Hickey MJ, Mathur SK, Zakel KL, Grotzke JE, Lewinsohn DM, Smith S, Sherman DR (2004) Individual RD1-region genes are required for export of ESAT-6/CFP-10 and for virulence of Mycobacterium tuberculosis. Mol Microbiol 51:359-370

Gupta S, Sinha A, Sarkar D (2006) Transcriptional autoregulation by Mycobacterium tuberculosis PhoP involves recognition of novel direct repeat sequences in the regulatory region of the promoter. FEBS Lett 580:5328-5338

Hsu T, Hingley-Wilson SM, Chen B, Chen M, Dai AZ, Morin PM, Marks CB, Padiyar J, Goulding C, Gingery M, Eisenberg D, Russell RG, Derrick SC, Collins FM, Morris SL, King CH, Jacobs WR Jr (2003) The primary mechanism of attenuation of bacillus Calmette-Guerin is a loss of secreted lytic function required for invasion of lung interstitial tissue. Proc Natl Acad Sci U S A 100:12420-12425

Huard RC, Fabre M, De Haas P, Lazzarini LCO, Van Soolingen D, Cousins D, Ho JL (2006) Novel genetic polymorphisms that further delineate the phylogeny of the Mycobacterium tuberculosis complex. J Bacteriol 188:4271-4287

Hunt DM, Saldanha JW, Brennan JF, Benjamin P, Strom M, Cole JA, Spreadbury CL, Buxton RS (2008) Single nucleotide polymorphisms that cause structural changes in the cyclic AMP receptor protein 
transcriptional regulator of the tuberculosis vaccine strain Mycobacterium bovis BCG alter global gene expression without attenuating growth. Infect Immun 76:2227-2234

Imaeda T, Coppola KM, Furness G (1985) Deoxyribonucleic acids of Corynebacterium genitalium and Corynebacterium pseudogenitalium: their genome molecular weights, base ratios, and DNA relatedness with other corynebacteria involved in urinary tract infections. Can J Microbiol 31:1068-1070

Kaufmann SHE, Winau F (2005) From bacteriology to immunology: the dualism of specificity. Nat Immunol 6:1063-1066

Kaufmann SHE, Hussey G, Lambert PH (2010) New vaccines for tuberculosis. Lancet 375:2110-2119

Keating LA, Wheeler PR, Mansoor H, Inwald JK, Dale J, Hewinson RG, Gordon SV (2005) The pyruvate requirement of some members of the Mycobacterium tuberculosis complex is due to an inactive pyruvate kinase: implications for in vivo growth. Mol Microbiol 56:163-174

Keller PM, Bottger EC, Sander P (2008) Tuberculosis vaccine strain Mycobacterium bovis BCG Russia is a natural recA mutant. BMC Microbiol 8:120

Kolibab K, Derrick SC, Morris SL (2011) Sensitivity to isoniazid of Mycobacterium bovis BCG strains and BCG disseminated disease isolates. J Clin Microbiol 49:2380-2381

Kozak R, Behr MA (2011) Divergence of immunologic and protective responses of different BCG strains in a murine model. Vaccine 29:1519-1526

Kozak RA, Alexander DC, Liao R, Sherman DR, Behr MA (2011) Region of difference 2 contributes to virulence of Mycobacterium tuberculosis. Infect Immun 79:59-66

Ladefoged A, Bunch-Christensen K, Guld J (1976) Tuberculin sensitivity in guinea-pigs after vaccination with varying doses of BCG of 12 different strains. Bull World Health Organ 53:435-443

Leung AS, Tran V, Wu Z, Yu X, Alexander DC, Gao GF, Zhu B, Liu J (2008) Novel genome polymorphisms in $\mathrm{BCG}$ vaccine strains and impact on efficacy. BMC Genomics 9:413

Lewis KN, Liao R, Guinn KM, Hickey MJ, Smith S, Behr MA, Sherman DR (2003) Deletion of RD1 from Mycobacterium tuberculosis mimics bacille CalmetteGuerin attenuation. J Infect Dis 187:117-123

Lind A (1983) The Swedish strain of Bcg. Tubercle 64:233-234

Liu J, Tran V, Leung AS, Alexander DC, Zhu B (2009) BCG vaccines: their mechanisms of attenuation and impact on safety and protective efficacy. Hum Vaccin 5:70-78

Lotte A, Wasz-Hockert O, Poisson N, Dumitrescu N, Verron M, Couvet E (1984) BCG complications. Estimates of the risks among vaccinated subjects and statistical analysis of their main characteristics. Adv Tuberc Res 21:107-193

Mahairas GG, Sabo PJ, Hickey MJ, Singh DC, Stover CK (1996) Molecular analysis of genetic differences between Mycobacterium bovis BCG and virulent Mbovis. J Bacteriol 178:1274-1282

Matsunaga I, Bhatt A, Young DC, Cheng TY, Eyles SJ, Besra GS, Briken V, Porcelli SA, Costello CE, Jacobs WR Jr, Moody DB (2004) Mycobacterium tuberculosis pks12 produces a novel polyketide presented by CD1c to T cells. J Exp Med 200:1559-1569

Mcshane H (2011) Tuberculosis vaccines: beyond bacille Calmette-Guerin. Philos Trans R Soc Lond Ser B Biol Sci 366:2782-2789

Milstien JB, Gibson JJ (1990) Quality control of BCG vaccine by WHO: a review of factors that may influence vaccine effectiveness and safety. Bull World Health Organ 68:93-108

Mostowy S, Tsolaki AG, Small PM, Behr MA (2003) The in vitro evolution of BCG vaccines. Vaccine 21:42704274

Munoz ME, Ponce E (2003) Pyruvate kinase: current status of regulatory and functional properties. Comp Biochem Physiol B Biochem Mol Biol 135:197-218

Naka T, Maeda S, Niki M, Ohara N, Yamamoto S, Yano I, Maeyama J, Ogura H, Kobayashi K, Fujiwara N (2011) Lipid phenotype of two distinct subpopulations of Mycobacterium bovis Bacillus Calmette-Guerin Tokyo 172 substrain. J Biol Chem 286:44153-44161

Obayashi Y (1955) Dried BCG vaccine. Monogr Ser World Health Organ, 1-220

Oettinger T, Jorgensen M, Ladefoged A, Haslov K, Andersen P (1999) Development of the Mycobacterium bovis BCG vaccine: review of the historical and biochemical evidence for a genealogical tree. Tuber Lung Dis 79:243-250

Ottenhoff TH (2009) Overcoming the global crisis: "yes, we can", but also for TB ... ? Eur J Immunol 39:20142020

Ottenhoff THM, Kaufmann SHE (2012) Vaccines against tuberculosis: where are we and where do we need to go? PLoS Pathog 8

Parish T, Smith DA, Kendall S, Casali N, Bancroft GJ, Stoker NG (2003) Deletion of two-component regulatory systems increases the virulence of Mycobacterium tuberculosis. Infect Immun 71:1134-1140

Pelayo MCG, Uplekar S, Keniry A, Lopez PM, Garnier T, Garcia JN, Boschiroli L, Zhou XM, Parkhill J, Smith N, Hewinson RG, Cole ST, Gordon SV (2009) A comprehensive survey of single nucleotide polymorphisms (SNPs) across Mycobacterium bovis strains and M. bovis BCG vaccine strains refines the genealogy and defines a minimal set of SNPs that separate virulent M. bovis strains and $M$. bovis BCG strains. Infect Immun 77:2230-2238

Pym AS, Brodin P, Majlessi L, Brosch R, Demangel C, Williams A, Griffiths KE, Marchal G, Leclerc C, Cole ST (2003) Recombinant BCG exporting ESAT-6 confers enhanced protection against tuberculosis. Nat Med 9:533-539

Rosenthal SR, Loewinsohne, Graham ML, Liveright D, Thorne G, Johnson V (1961) BCG vaccination against tuberculosis in Chicago. A twenty-year study statistically analyzed. Pediatrics 28:622-641 
Sakula A (1983) BCG: who were Calmette and Guerin? Thorax 38:806-812

Salamon H, Kato-Maeda M, Small PM, Drenkow J, Gingeras TR (2000) Detection of deleted genomic DNA using a semiautomated computational analysis of GeneChip data. Genome Res 10:2044-2054

Sherman DR, Guinn KM, Hickey MJ, Mathur SK, Zakel KL, Smith S (2004) Mycobacterium tuberculosis H37Rv: Delta RD1 is more virulent than M. bovis bacille Calmette-Guerin in long-term murine infection. J Infect Dis 190:123-126

Singh A, Crossman DK, Mai D, Guidry L, Voskuil MI, Renfrow MB, Steyn AJ (2009) Mycobacterium tuberculosis WhiB3 maintains redox homeostasis by regulating virulence lipid anabolism to modulate macrophage response. PLoS Pathog 5:e1000545

Sinha A, Gupta S, Bhutani S, Pathak A, Sarkar D (2008) PhoP-PhoP interaction at adjacent PhoP binding sites is influenced by protein phosphorylation. $\mathrm{J}$ Bacteriol 190:1317-1328

Stanley SA, Raghavan S, Hwang WW, Cox JS (2003) Acute infection and macrophage subversion by Mycobacterium tuberculosis require a specialized secretion system. Proc Natl Acad Sci U S A 100:1300113006

Stewart GR, Snewin VA, Walzl G, Hussell T, Tormay P, O'Gaora P, Goyal M, Betts J, Brown IN, Young DB (2001) Overexpression of heat-shock proteins reduces survival of Mycobacterium tuberculosis in the chronic phase of infection. Nat Med 7:732-737

Stewart GR, Wernisch L, Stabler R, Mangan JA, Hinds J, Laing KG, Young DB, Butcher PD (2002) Dissection of the heat-shock response in Mycobacterium tuberculosis using mutants and microarrays. Microbiology 148:3129-3138

Steyn AJ, Collins DM, Hondalus MK, Jacobs WR Jr, Kawakami RP, Bloom BR (2002) Mycobacterium tuberculosis WhiB3 interacts with RpoV to affect host survival but is dispensable for in vivo growth. Proc Natl Acad Sci U S A 99:3147-3152

Takayama K, Wang L, David HL (1972) Effect of isoniazid on the in vivo mycolic acid synthesis, cell growth, and viability of Mycobacterium tuberculosis. Antimicrob Agents Chemother 2:29-35

Tran V, Ahn SK, Ng M, Li M, Liu J (2016) Loss of lipid virulence factors reduces the efficacy of the BCG vaccine. Sci Rep 6:29076

Trunz BB, Fine P, Dye C (2006) Effect of BCG vaccination on childhood tuberculous meningitis and miliary tuberculosis worldwide: a meta-analysis and assessment of cost-effectiveness. Lancet 367:1173-1180

Vallishayee RS, Shashidhara AN, Bunch-Christensen K, Guld J (1974) Tuberculin sensitivity and skin lesions in children after vaccination with 11 different BCG strains. Bull World Health Organ 51:489-494

Wallgren A (1928) Intradermal vaccinations with BCG virus - preliminary note. J Am Med Assoc 91:18761881

Walters SB, Dubnau E, Kolesnikova I, Laval F, Daffe M, Smith I (2006) The Mycobacterium tuberculosis PhoPR two-component system regulates genes essential for virulence and complex lipid biosynthesis. Mol Microbiol 60:312-330

Wang S, Engohang-Ndong J, Smith I (2007) Structure of the DNA-binding domain of the response regulator PhoP from Mycobacterium tuberculosis. Biochemistry 46:14751-14761

Wernisch L, Kendall SL, Soneji S, Wietzorrek A, Parish T, Hinds J, Butcher PD, Stoker NG (2003) Analysis of whole-genome microarray replicates using mixed models. Bioinformatics 19:53-61

Wiker HG, Nagai S, Hewinson RG, Russell WP, Harboe M (1996) Heterogenous expression of the related MPB70 and MPB83 proteins distinguish various substrains of Mycobacterium bovis BCG and Mycobacterium tuberculosis H37Rv. Scand J Immunol 43:374380

Zwaig N, Kistler WS, Lin EC (1970) Glycerol kinase, the pacemaker for the dissimilation of glycerol in Escherichia coli. J Bacteriol 102:753-759

Zwerling A, Behr MA, Verma A, Brewer TF, Menzies D, Pai M (2011) The BCG world atlas: a database of global BCG vaccination policies and practices. PLoS Med 8:e1001012 